\title{
Massive juvenile ossifying fibroma of maxillary sinus with orbital involvement
}

\author{
JERRY A SHIELDS,' ROBERT G PEYSTER, ${ }^{2}$ STEVEN D HANDLER, ${ }^{3}$ \\ JAMES J AUGSBURGER, ' AND JAMES KAPUSTIAK' ${ }^{1}$
}

From the 'Oncology Service, Wills Eye Hospital, Jefferson Medical College, Thomas Jefferson University, Philadelphia; the ${ }^{2}$ Department of Radiology, Neurology, and Neurosurgery, Hahnemann University, Philadelphia; and the ${ }^{3}$ Department of Otolaryngology, Children's Hospital of Philadelphia and the University of Pennsylvania School of Medicine, Philadelphia, USA

SUMmARY A 14-year-old black girl had painless, progressive proptosis and upward displacement of the left eye for approximately six months. Computed tomography showed a massive tumour involving the maxillary sinus with extension into the sphenoid sinus, ethmoid sinus, and orbit. The mass was excised surgically and proved histopathologically to be a juvenile ossifying fibroma. The clinical and histopathological features of this uncommon orbital tumour are discussed and its place in the spectrum of fibro-osseous tumours of the orbital region is considered.

Ossifying fibroma is a rather uncommon fibroosseous tumour which can involve the bones of the orbit and paranasal sinuses and produce proptosis. This tumour has generally been difficult to differentiate clinically and histopathologically from fibrous dysplasia and other fibro-osseous tumours of the orbital region. ${ }^{12}$ Recent publications, however, have pointed out that it does have certain clinical and histopathological features which serve to differentiate it from other related lesions. ${ }^{34}$ Some authors have reported that computed tomography can be particularly helpful in diagnosing ossifying fibroma. ${ }^{4}$ Most ossifying fibromas in the orbital area have arisen from the orbital plate of the frontal bone or from the ethmoid sinus region, and ossifying fibromas of the maxillary sinus with secondary orbital involvement appear to be quite rare. ${ }^{5} \mathrm{We}$ report here the case of 14-year-old girl who developed proptosis and upward displacement of the left eye secondary to a massive juvenile ossifying fibroma which arose from the maxillary sinus and secondarily invaded the orbit.

\section{Case report}

A 14-year-old black girl with no prior ocular or systemic problems developed slowly progressive proptosis and upward displacement of the left eye

Correspondence to Dr J A Shields, Oncology Service, Wills Eye Hospital, Ninth and Walnut Streets, Philadelphia, PA 19107, USA. over a six-month period. She was referred to the Oncology Service of Wills Eye Hospital for examination and treatment.

Ocular examination disclosed a visual acuity of $6 / 5$ $(20 / 15)$ in the right eye and 6/7.5 (20/15) in the left eye. The intraocular pressure was $11 \mathrm{Hgmm}$ in both eyes by applanation tonometry. There was obvious proptosis, with upward displacement of the left eye (Fig. 1). There was a slight left exotropia on upgaze and slight limitation of supraduction with the left eye. Hertel exophthalmometry revealed $8 \mathrm{~mm}$ of left proptosis, with readings of 18 and 26 for the right and left eyes respectively. No orbital masses could be palpated. Fundus examination revealed no indentation of the globe by an orbital mass and no choroidal folds. Ultrasonography disclosed a large posterior orbital mass with high internal reflectivity.

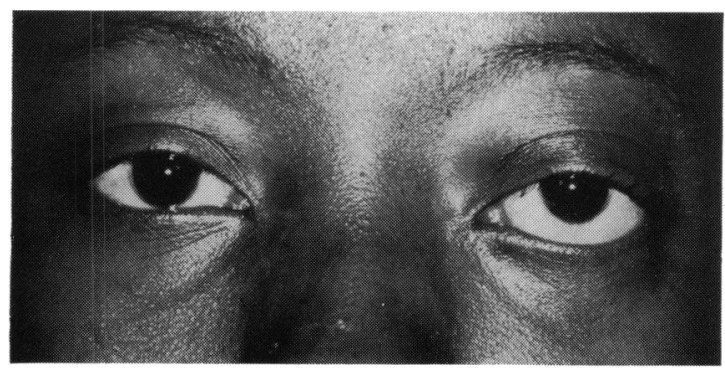

Fig. 1 Clinical photograph showing upward displacement of the left eye with proptosis. 


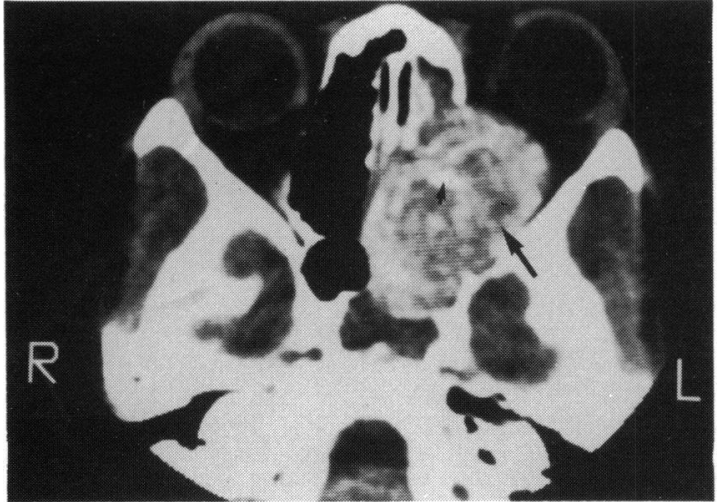

Fig. 2A Axial section without contrast material at the level of the inferior orbits shows a large, well circumscribed hyperdense mass (large arrow) involving the left ethmoid, upper maxillary, and sphenoid sinuses, with extension well into the left orbit. There are numerous foci of calcification or ossification within the lesion, the most prominent of which is indicated (small arrow). Destruction of the bony margins of the involved sinuses is noted.

Computed tomography (CT) without contrast material in axial and coronal planes showed a large, well circumscribed mass filling the left maxillary sinus and extending into the left orbit, ethmoid, and sphenoid sinuses, the nasal cavity, and intracranially to the infrafrontal epidural space (Figs. 2A, B, C). The lesion was nonhomogeneous owing to foci of ossification and was predominantly dense relative to brain. Frank bone destruction was noted in several sites, while in other areas bone was displaced around the edge of the lesion. Exophthalmos was noted on the left side.

On the basis of the clinical and CT findings our clinical diagnosis was a fibro-osseous lesion, most

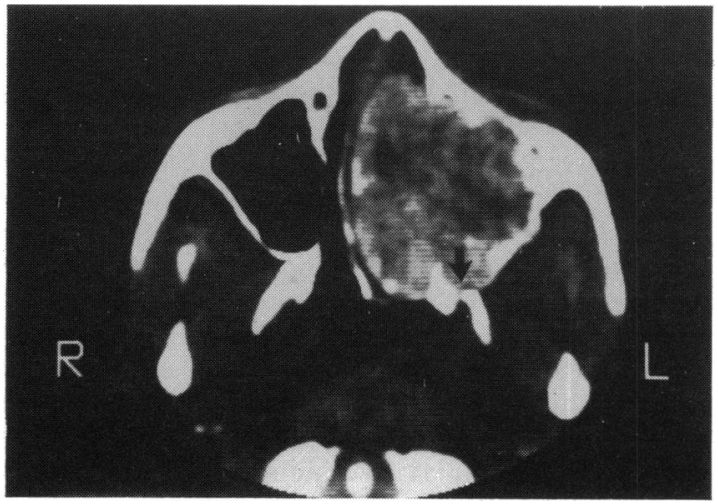

Fig. 2B Axial CT inferior to that shown in Fig. 2A. The mass fills the left maxillary sinus and most of the left nasal cavity. Destruction of the medial wall of the maxillary sinus and of the left pterygoid bone (arrow) are seen.

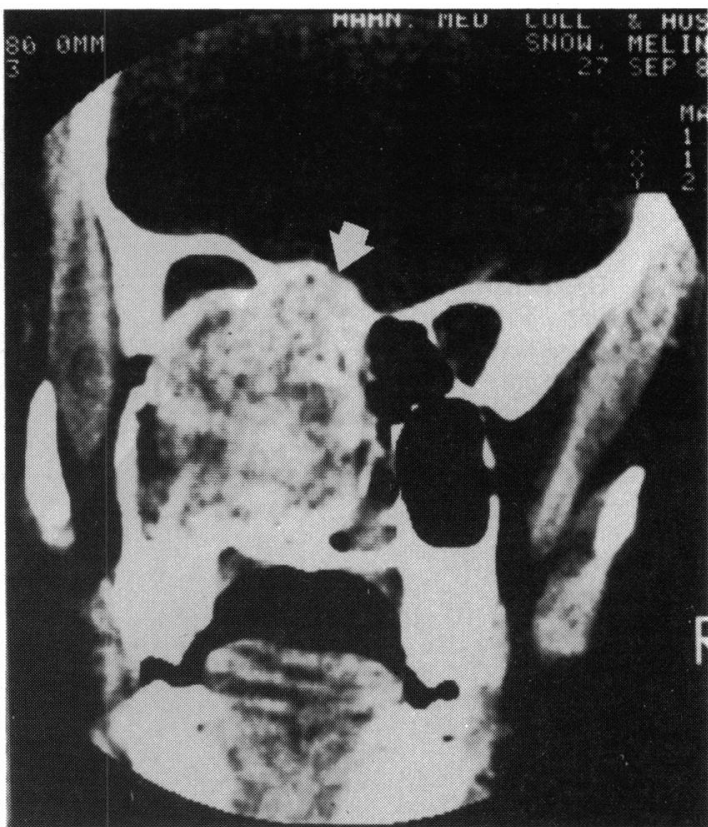

Fig. 2C Coronal CT without contrast material at the level of the posterior orbits. (Note that the patient's left side corresponds with the reader's in the coronal view.) The large mass fills the left maxillary and ethmoid sinuses, the left nasal cavity, and has invaded the infrafrontal intracranial epidural space (arrow). The heterogeneous density of the lesion, with many foci of calcification and/or ossification, is well shown. The mass has bowed the left orbital floor superiorly.

likely ossifying fibroma. Under general anaesthesia a Lynch incision was made and extended inferiorly to expose the medial aspect of the maxillary wall. The periosteum was elevated and the thinned anterior maxillary wall was exposed. The large size and unyielding nature of the mass made removal in one piece impossible. Therefore the gritty tumour was removed piecemeal. After complete removal of the mass, which extended into the ethmoid and sphenoid sinuses, the cavity was irrigated and packed with antibiotic-coated new gauze. Blood loss was less than $150 \mathrm{ml}$ and there were no surgical complications. The patient was doing well, with good vision and no systemic or ocular problems approximately three months after the surgery.

\section{PATHOLOGICAL FINDINGS}

The specimen consisted of several pieces of rather gritty, tan-brown tissue ranging in size from $11 \times 8 \times 3.5 \mathrm{~cm}$ to $0.4 \times 0.2 \times 0.2 \mathrm{~cm}$. Histological sections showed it to consist of a cellular, fibroblastic stroma containing spindle-shaped cells and numerous osseous spicules. A few giant cells were present adjacent to the bony spicules. On higher-power 
Fig. 3 Photomicrographshowing spicules of bone (arrow) with surrounding osteoid and a rim of osteoblasts around the osteoid material outside the bony spicules. $A$ giant cell is present adjacent to the bony spicule. (Haematoxylin and eosin, $\times$ 67).

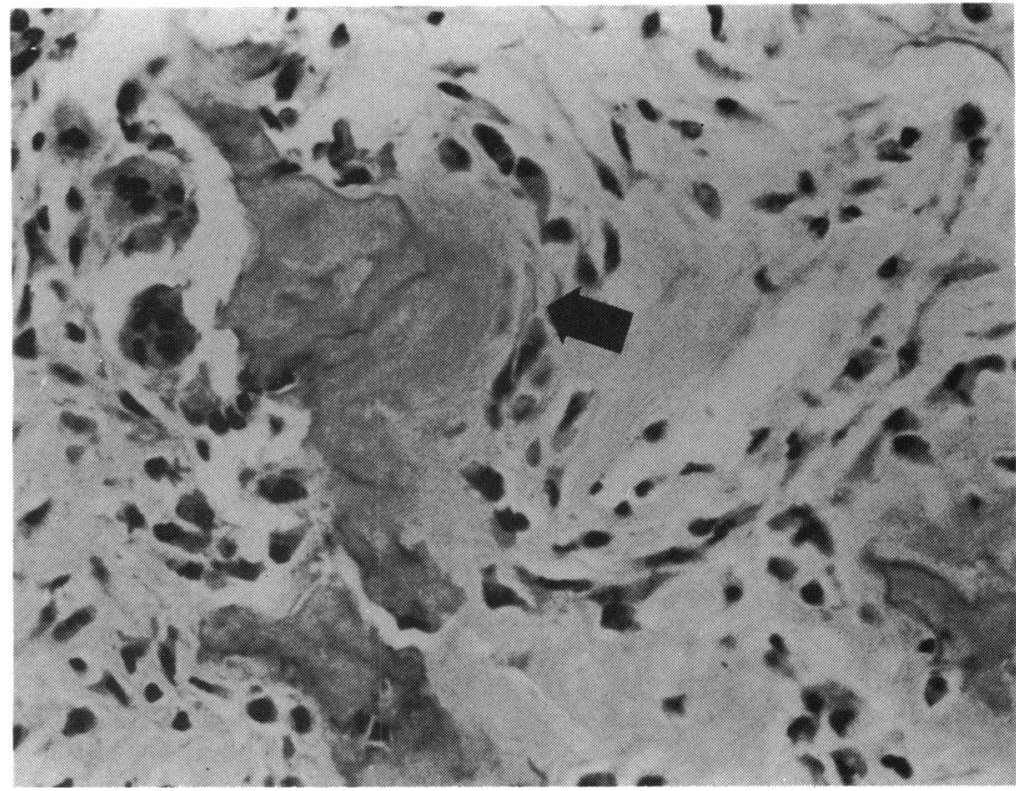

microscopy it was noted that the bony trabeculae were lined by lightly eosinophilic material, suggestive of osteoid, which was in turn rimmed by osteoblasts (Fig. 3). These findings were compatible with the diagnosis of ossifying fibroma of bone.

\section{Discussion}

The classification of fibro-osseous tumours, particularly those in the orbital region, has been the subject of some controversy. ${ }^{2}$ Blodi has stated that ossifying fibromas in the cranial region might be regarded as a variant of fibrous dysplasia.' Other authorities have expressed the opinion that ossifying fibromas should be grouped as a variant under the more general category of osteoblastomas. ${ }^{2}$ More recently other authors have believed that the histopathological findings are sufficient to classify ossifying fibromas into a separate category..$^{3-9} \mathrm{~A}$ recent report has delineated the clinical, histopathological, and computed tomographic characteristics which help in the diagnosis of juvenile ossifying fibroma. ${ }^{4}$ The CT finding of a well circumscribed tumour with patchy areas of sclerosis within the matrix of the lesion strongly suggested the diagnosis of ossifying fibroma in the case reported here. Margo and associates recently reviewed the cases of ossifying fibromas with orbital involvement from the files of the Armed Forces Institute of Pathology. ${ }^{5}$ Among 21 cases on file 10 lesions were centred in the orbital plate of the frontal bone, nine in the ethmoid sinus, and in two cases the precise location was not determined. These tumours characteristically produced downward or lateral displacement of the globe. Our patient was particularly unusual in that the tumour produced proptosis and upward displacement of the globe, indicating its origin in the maxillary sinus. Ossifying fibroma of the maxillary sinus should be considered in the differential diagnosis when a child or young adult presents with proptosis and slowly progressive upward displacement of the globe.

Although ossifying fibroma is generally believed to be difficult to diagnose histopathologically, Margo and associates have recently presented a detailed account of the rather distinctive features of this tumour as seen by light and electron microscopy. ${ }^{5}$ The numerous rounded, mineralised collagenous foci, which they termed 'psammomatoid ossicles,' were the basis for their favouring the term 'psammomatoid ossifying fibroma.'

It is currently believed that the best management of juvenile ossifying fibromas is surgical excision. Because these tumours can recur after incomplete excision, ${ }^{5}$ every attempt should be made to remove the mass completely. In most cases this must be accomplished piecemeal to remove as much of the mass as possible while protecting adjacent vital and cosmetically important structures. The surgical approach should be determined by the location and size of the tumour as indicated by CT. Computed tomography is also invaluable in the postoperative follow-up to detect recurrence of the tumour.

Although the pathogenesis of juvenile ossifying fibroma is unknown, it appears to be a spontaneously 
occurring benign neoplasm of bone, which most commonly becomes clinically apparent in children and young adults between the ages of 6 and 21 years. ${ }^{5}$ We believe that clinical awareness of this tumour, combined with further refinement of its CT features, will lead to more accurate clinical diagnosis and more effective management of patients with juvenile ossifying fibromas in the orbital region.

The patient was also evaluated clinically by Dr Lois Martyn. Mr David Silva also provided assistance.

This work was supported in part by the Pennsylvania Lions Sight Conservation and Eye Research Foundation, Inc., the Ocular Oncology Fund, and the Oncology Research Fund, Wills Eye Hospital, Philadelphia, USA.

\section{References}

1 Blodi FC. Pathology of the orbital bones. The XXXII Edward Jackson Memorial Lecture. Am J Ophthalmol 1976; 81: 1-26.
2 Henderson JW. Orbital tumors. 2nd ed. New York: Decker, 1980: 205-8, 225-31.

3 Jones IS, Jakobiec FJ, eds. Diseases of the orbit. Hagerstown: Harper and Row, 1979: 483-502.

4 Shields JA, Nelson LB, Brown JF, Dolinskas C. Clinical, computed tomographic, and histopathologic characteristics of juvenile ossifying fibroma with orbital involvement. $A m J$ Ophthalmol 1983; 96: 650-3.

5 Margo CE, Ragsdale BD, Perman KI, Zimmerman LE, Sweet DE. Psammomatoid (juvenile) ossifying fibroma of the orbit. Ophthalmology (Rochester) in press.

6 Dahlin DC. Bone tumors. General aspects and data on 621 cases. Springfield: Thomas, 1978: 362-9.

7 Scott M, Peale AR, Croissant PD. Intracranial midline anterior fossae ossifying fibroma invading orbits, paranasal sinuses, and right maxillary antrum. $J$ Neurosurg 1971; 34: 827-31.

8 Kahlil MK, Leib ML. Cemento-ossifying fibroma of the orbit. Can J Ophthalmol 1979; 14: 195-200.

9 Fu YS, Perzin KH. Non-epithelial tumors of the nasal cavity, paranasal sinuses, and nasopharynx. A clinicopathologic study. II. Osseous and fibro-osseous lesions, including osteoma, fibrous dysplasia, ossifying fibroma, osteoblastoma giant cell tumor, and osteosarcoma. Cancer 1974; 33: 1289-305. 